\title{
Clinical evaluation of St Jude Medical Hemodynamic Plus versus standard aortic valve prostheses: The Italian multicenter, prospective, randomized study
}

Nicola Vitale, MD, PhD

Ilaria Caldarera, MD

Claudio Muneretto, MD

Riccardo Sinatra, MD

Antonio Scafuri, MD

Elio Di Rosa, MD

Andrea Contini, MD

Nicola Tedesco, PhD

Angelo Pierangeli, MD

Mauro Abbate, MD

Tiziano Gherli, MD

Dino Casarotto, MD

Michele Di Summa, MD

Benedetto Marino, MD

Luigi Chiariello, MD

Luigi de Luca T.S., MD

See related editorial on page 645 .

From the Italian Multicenter Study Group for the St Jude Medical Hemodynamic Plus Aortic Valve Prosthesis.

Received for publication Aug 11, 2000; revisions requested Oct 23, 2000; revisions received Jan 23, 2001; accepted for publication Feb 20, 2001.

Address for reprints: Nicola Vitale MD, $\mathrm{PhD}$, Istituto di Cardiochirurgia, Policlinico, Piazza Giulio Cesare 11, Bari 70124, Italy (E-mail: Nicola.Vitale@excite.co.uk).

J Thorac Cardiovasc Surg 2001;122:691-8

Copyright (c) 2001 by The American Association for Thoracic Surgery

0022-5223/2001 \$35.00+ $0 \quad \mathbf{1 2 / 1 / 1 1 6 2 0 5}$

doi:10.1067/mtc.2001.116205
Objective: Hemodynamic and clinical performances of 21-mm and 23-mm St Jude Medical Hemodynamic Plus aortic valves (St Jude Medical, Inc, St Paul, Minn) were compared with those of 21-mm and 23-mm St Jude Medical standard cuff aortic valves in the first such multicenter, prospective, randomized study. Hemodynamic Plus valves are mechanical, bileaflet prostheses suitable for the small aortic anulus.

Methods: Patients with 21-mm and 23-mm anulus diameters were randomized to receive either a Hemodynamic Plus or a standard cuff valve. Postoperatively and at 6 months after the operation, patients underwent 2-dimensional Doppler echocardiography. Ejection fraction, cardiac output, peak gradient, mean gradient, effective orifice area, effective area index, and performance index were calculated. Postoperative and 6-month echocardiographic measurements and their variations across observation times were analyzed statistically.

Results: Of the 140 patients enrolled, 5 died at operation and 1 died of aortic dissection during the follow-up period. Eight patients were lost to follow-up. A total of 125 patients completed the study. In 1 patient a sewing cuff escaped intraoperatively. At 6 months the 21-mm and 23-mm Hemodynamic Plus valves showed significantly lower peak gradients and mean gradients than those of the 21-mm and 23$\mathrm{mm}$ standard cuff valves. The 21-mm Hemodynamic Plus valves had gradients similar to those of the 23-mm Hemodynamic Plus valves. The effective orifice area did not differ significantly between the Hemodynamic Plus and standard cuff valves at either measurement. No valve mismatch was found in the 4 groups of patients. A more enhanced decrease of peak gradients and mean gradients and a more enhanced increase of effective orifice areas, effective area indices, and performance indices were found across observation times for patients with Hemodynamic Plus valves compared with those with standard cuff valves. 
Conclusions: Clinical hemodynamic performances of 21-mm and 23-mm St Jude Medical Hemodynamic Plus valves correspond closely with those of standard cuff valves, and gradients are substantially better than those of standard cuff valves of the same diameter. Therefore, use of this valve may minimize the need for aortic anulus enlargement. Early follow-up results with the Hemodynamic Plus valves were excellent, although more time is required to confirm this outcome.

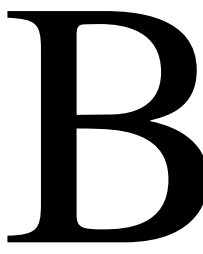

ileaflet mechanical replacement heart valves account for approximately 50\% of all heart valve prostheses currently implanted. ${ }^{1}$ The mechanical or hemodynamic function of bileaflet valves is considered to be good and acceptable for most patients. Nevertheless, concerns remain about the hemodynamic performances of bileaflet valves implanted in the small aortic anulus. ${ }^{1}$

To address this issue, St Jude Medical (St Paul, Minn) has introduced a new model of mechanical bileaflet heart valve suitable for the small aortic anulus-the St Jude Medical Hemodynamic Plus (HP) aortic valve. In this new valve, the sewing cuff has been reconfigured to allow supraannular placement. Eliminating the sewing cuff from the anulus achieves an effectively larger valve orifice with an equivalent tissue anulus diameter.

The Italian multicenter, prospective, randomized study was undertaken to evaluate the hemodynamic and clinical performances of the St Jude Medical HP valve in the aortic position by comparing it with the St Jude Medical standard cuff (SC) valve. This was the first study of its kind.

This report presents the results of this controlled study, providing an in vivo demonstration of the laboratoryenhanced hemodynamic characteristics of the HP valve and an assessment of early follow-up results with this valve.

\section{Methods}

This controlled study was undertaken by 8 Italian academic units of cardiac surgery. Study enrollment was 140 patients.

Admission criteria allowed patients with isolated aortic valve disease (no concomitant mitral, tricuspid, or pulmonic disease) requiring valve replacement who were also candidates for a mechanical valve prosthesis. Patients with other associated cardiac disease, such as coronary artery disease, were not eliminated.

Exclusion criteria eliminated patients requiring aortic valve replacement on an emergency basis, which did not permit the performance of complete preoperative investigations; patients with preoperative evaluations indicating other significant cardiovascular abnormalities, such as aortic aneurysm or dissection, left ventricular aneurysm, or active endocarditis; as well as patients with a life expectancy less than 2 years or with chronic liver or kidney failure.

The enrollment period lasted 1 year, and the follow-up period required for each patient was 6 months. Patients not monitored at the end of the study were considered lost for the considered outcome.
The trial protocol was approved by the institutional review board/ethics committee of each individual participating center. Informed consent was obtained from each patient before preoperative investigations.

\section{Study Protocol}

Patients enrolled were stratified by the model and size of valve prosthesis they received at operation (21-mm HP, 23-mm HP, 21mm SC, or 23-mm SC valve) and were studied accordingly.

Each patient underwent routine preoperative investigations. For the purposes of this study, postoperative (within 30 days after operation) and 6-month assessments included a clinical examination and a transthoracic echocardiogram. The oral anticoagulation level was kept within an international normalized ratio range of 2.5 and 3.5 in all participating centers.

For each patient, clinical conditions after each visit, echocardiographic data, anticoagulation status, and any mortal or morbid event were recorded on forms for purposes of statistical analysis. Any complication or death was registered at the time of occurrence and according to the relevant guidelines for valve surgery. ${ }^{2}$

\section{Patient Randomization}

In the operating theater, once the aorta was opened and the aortic valve excised, the anulus was sized. Patients with a 21-mm or 23$\mathrm{mm}$ anulus size were randomized to receive a St Jude Medical HP or SC valve. Randomization was carried out in a blind manner for both the patient and surgeon, by opening a sealed opaque envelope containing the assigned valve type. Envelopes contained random numbers that were generated by a computer program and were balanced every 4 patients.

\section{Surgical Technique}

All operations were performed by means of standard cardiopulmonary bypass with mild hypothermia. Myocardial protection was achieved with cold blood antegrade cardioplegia. In patients with aortic insufficiency, cardioplegic solution was infused directly into the coronary ostia.

After valve excision, the aortic anulus was completely debrided of calcium in all patients. All valves were sized with standard sizers. If randomization assigned an HP valve, the aortic anulus was sized again with the HP sizer. A close correspondence between the standard and HP sizers was observed for both 21-mm and 23-mm valve sizes.

All HP valves were implanted at the supra-annular level with interrupted everting pledget-supported mattress sutures. Each valve was oriented with its axis perpendicular to the septum. All the SC valves were implanted at the infra-annular level with the same implantation technique and valve orientation. 
TABLE 1. Patient characteristics

\begin{tabular}{|c|c|c|c|c|}
\hline Parameter & 21-mm SC & 21-mm HP & 23-mm SC & 23-mm HP \\
\hline $\mathrm{N}$ & 35 & 35 & 35 & 35 \\
\hline Age (y) & $63.6 \pm 8$ & $61.3 \pm 10$ & $60.6 \pm 10$ & $57 \pm 15$ \\
\hline \multicolumn{5}{|l|}{ Sex } \\
\hline$M$ & 14 & 20 & 22 & 27 \\
\hline $\mathrm{F}$ & 21 & 15 & 13 & 8 \\
\hline Aortic stenosis & 24 & 24 & 12 & 17 \\
\hline Stenosis + regurgitation & 11 & 7 & 11 & 9 \\
\hline Regurgitation & - & 4 & 12 & 9 \\
\hline \multicolumn{5}{|l|}{ NYHA } \\
\hline I & - & 1 & 2 & - \\
\hline II & 14 & 14 & 18 & 15 \\
\hline III & 21 & 20 & 12 & 16 \\
\hline IV & - & - & 3 & 4 \\
\hline Body surface area $\left(\mathrm{m}^{2}\right)$ & $1.72 \pm 0.18$ & $1.74 \pm 0.15$ & $1.78 \pm 0.21$ & $1.78 \pm 0.15$ \\
\hline CPB time (min) & $99.5 \pm 0.18$ & $107 \pm 35$ & $93.4 \pm 33$ & $84.3 \pm 25$ \\
\hline Crossclamping time (min) & $75.7 \pm 24$ & $76.4 \pm 24$ & $69 \pm 22$ & $64 \pm 16$ \\
\hline
\end{tabular}

$N$, Number of patients enrolled in the study; $S C$, St Jude Medical standard cuff valve; HP, St Jude Medical Hemodynamic Plus valve; NYHA, New York Heart Association; $C P B$, Cardiopulmonary bypass. There was no significant difference in age $(P=.22)$, body surface area $(P=.40)$, and crossclamping time $(P=$ .35). Difference in CPB time reached statistical significance $(P=.039)$.

Concomitant procedures were coronary artery bypass grafting in 10 cases, closure of a patent ductus arteriosus in 1 case, and atrial septal defect in 1 case.

\section{Echocardiography}

In each participating center, echocardiograms for the assessment of functional and hemodynamic valve performances were always carried out by the same echocardiographer who was unaware of the type of valve implanted.

Doppler echocardiography was obtained from apical and parasternal long-axis views. The ultrasonography window from which the highest velocities were obtained was selected and used for Doppler evaluation. Doppler approach was similar for all participating centers. Flow velocity in the left ventricular outflow tract and across the valve was measured by means of pulsed and continuous wave Doppler ultrasonography, respectively. The modified Bernoulli equation was used to calculate peak and mean pressure gradients across the prosthesis. Color Doppler ultrasonography was used to visualize and quantify the amount and site of prosthetic valve regurgitation.

\section{Doppler Measurements and Calculations}

All of the following parameters were collected from each patient:

Cardiac output $=$ time velocity integral $\times$ effective orifice area $\times$ heart rate.

Peak gradient $=4\left(\mathrm{~V}_{2}{ }^{2}-\mathrm{V}_{1}{ }^{2}\right)$, where $\mathrm{V}_{2}$ is prosthetic peak velocity in meters per second measured with continuous-wave Doppler ultrasonography and $V_{1}$ is peak velocity proximal to valve in meters per second, measured with pulsed-wave Doppler ultrasonography.
Mean gradient $=4\left[(\mathrm{VTI})_{2}{ }^{2}-(\mathrm{VTI})_{1}{ }^{2}\right]$, where $\mathrm{VTI}_{2}$ is prosthetic velocity time integral measured with continuous-wave Doppler ultrasonography and $\mathrm{VTI}_{1}$ is proximal velocity time integral measured with pulsed-wave Doppler ultrasonography, calculated by online averaging.

Effective orifice area. The effective orifice area (EOA) is an index of how well a valve design uses its geometric orifice area. It was calculated with the continuity equation by the simplified peak velocity method as CSA $\left(\mathrm{PkV}_{\mathrm{LVOT}} / \mathrm{PkV}_{\mathrm{Ao}}\right)$, where CSA is the subvalvular cross-sectional area and $\mathrm{PkV}_{\mathrm{LVOT}}$ and $\mathrm{PkV}_{\mathrm{Ao}}$ are the maximal velocity in the left ventricular outflow tract and across the valve, respectively. The cross-sectional area was calculated by measuring the left ventricular outflow tract just below the prosthetic ring by means of an edge-to-edge method.

Effective area index. The effective area index (EIA) is a measure of how well the EOA of the valve matches the body surface area (BSA). It is calculated as EAI = EOA/BSA. This index was used to detect mismatch between valve size and body surface area. According to Dumesnil and associates, ${ }^{3}$ it would appear that an indexed prosthetic valve area of approximately $0.9 \mathrm{~cm}^{2} / \mathrm{m}^{2}$ would be adequate to minimize the postoperative gradient.

Performance index. The performance index (PI) is a measure of how effectively the external dimension of the valve is used to provide forward flow, normalized to the valve size. It is defined as PI = EOA/geometric area. Most articles provide performance index, making possible the comparison with other valve types.

\section{Statistical Analysis}

As a first step, 1-way analysis of variance for unbalanced blocks was carried out to compare age, body surface area, aortic crossclamping time, and cardiopulmonary bypass time by means of the 4 valve types. 


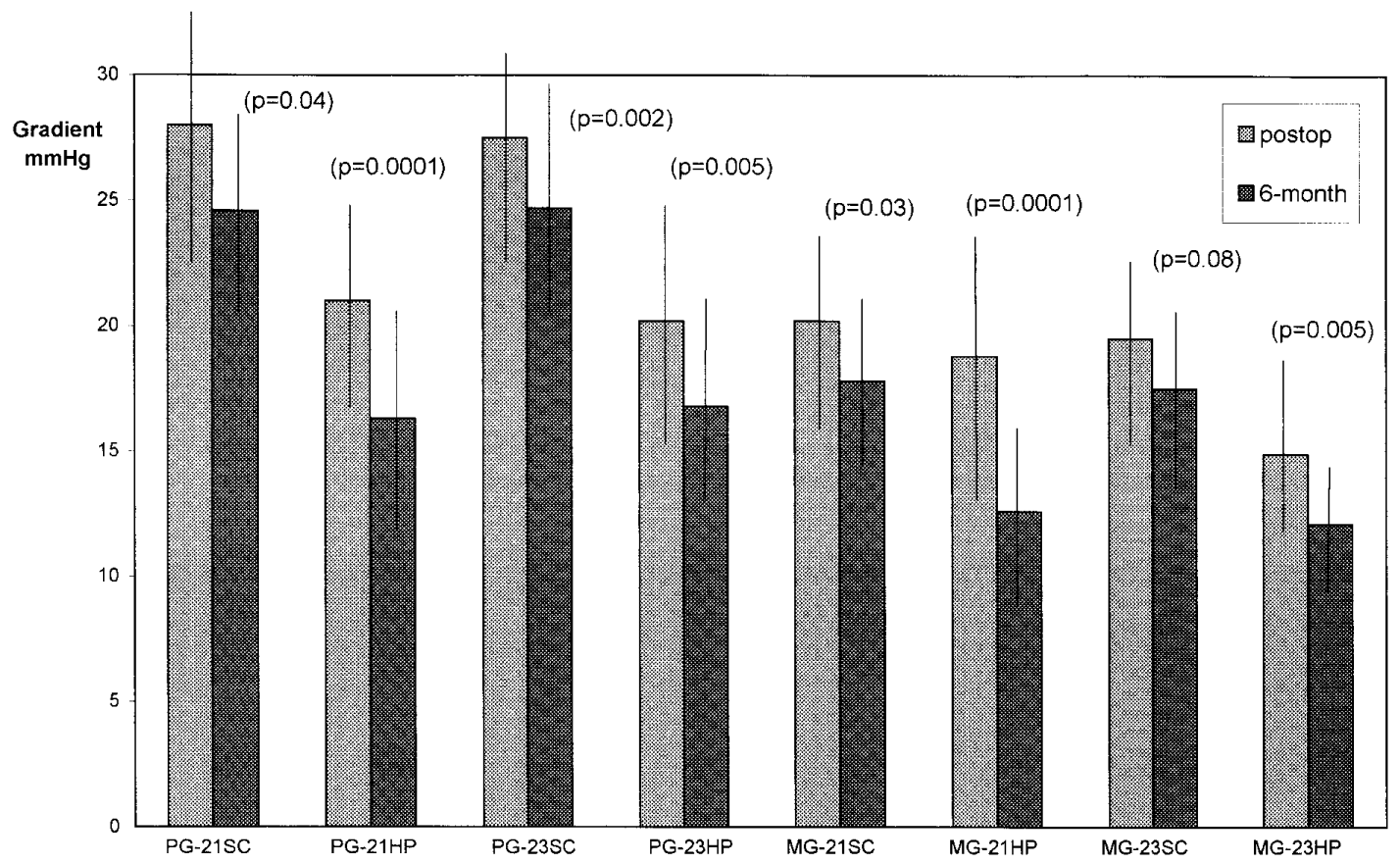

Figure 1. Serial changes of peak and mean transvalvular gradients over time within the 4 groups. The $P$ values express the comparisons between postoperative and 6-month observation times. MG, Mean gradients; $P G$, peak gradients.

TABLE 2. Postoperative echocardiographic data

\begin{tabular}{|c|c|c|c|c|c|c|c|}
\hline Parameter & $\begin{array}{c}21-\mathrm{mm} \mathrm{SC} \\
(\mathrm{n}=27)\end{array}$ & $P^{*}$ & $\begin{array}{c}\text { 21-mm HP } \\
(\mathrm{n}=35)\end{array}$ & $P \dagger$ & $\begin{array}{c}\text { 23-mm SC } \\
(\mathrm{n}=30)\end{array}$ & $P^{\ddagger}$ & $\begin{array}{c}\text { 23-mm HP } \\
(n=33)\end{array}$ \\
\hline $\mathrm{CO}(\mathrm{L} / \mathrm{m})$ & $5.84 \pm 1.8$ & .016 & $4.32 \pm 1.7$ & .0004 & $6.51 \pm 2.7$ & .019 & $4.97 \pm 1.9$ \\
\hline $\mathrm{EF}$ & $0.56 \pm 0.9$ & .51 & $0.54 \pm 0.9$ & .98 & $0.54 \pm 0.9$ & .67 & $0.53 \pm 0.1$ \\
\hline Peak gradient (mm Hg) & $28.0 \pm 9.9$ & .005 & $21.0 \pm 10$ & .0059 & $27.5 \pm 7.3$ & .0034 & $20.2 \pm 8.8$ \\
\hline Mean gradient $(\mathrm{mm} \mathrm{Hg})$ & $20.2 \pm 4.7$ & .5 & $18.8 \pm 10$ & .7 & $19.5 \pm 5.3$ & .025 & $14.9 \pm 5.3$ \\
\hline $\mathrm{EOA}\left(\mathrm{cm}^{2}\right)$ & $1.53 \pm 0.5$ & .2 & $1.56 \pm 0.4$ & .011 & $1.66 \pm 0.5$ & .72 & $1.60 \pm 0.6$ \\
\hline $\mathrm{EAI}\left(\mathrm{cm}^{2}\right)$ & $0.90 \pm 0.3$ & .074 & $0.90 \pm 0.2$ & .7 & $0.94 \pm 0.2$ & .69 & $0.90 \pm 0.3$ \\
\hline $\mathrm{PI}\left(\mathrm{cm}^{2}\right)$ & $0.64 \pm 0.2$ & .08 & $0.61 \pm 0.2$ & .8 & $0.64 \pm 0.2$ & .2 & $0.55 \pm 0.2$ \\
\hline
\end{tabular}

Data are expressed as mean \pm standard deviation. The number of patients in each group is indicated in parentheses. $S C$, St Jude Medical standard cuff valve; $H P$, St Jude Medical Hemodynamic Plus valve; $C O$, cardiac output; $E F$, ejection fraction; EOA, effective orifice area; $E A I$, effective area index; $P I$, performance index.

${ }^{*} P$ value, 21-mm SC vs 21-mm HP valves.

† $P$ value, $21-\mathrm{mm} \mathrm{HP}$ vs $23-\mathrm{mm} \mathrm{SC}$ valves.

$\ddagger P$ value, $23-\mathrm{mm} \mathrm{SC}$ vs $23-\mathrm{mm} \mathrm{HP}$ valves.

Second, analysis of variance was applied to study performance characteristics of the 4 valve types, separately in the 2 observation times (postoperatively and 6 months after the operation). In this framework, orthogonal comparisons were carried out for the 4 blocks and for linear contrasts regarding specific couplings (21$\mathrm{mm}$ HP vs 21-mm SC; $21-\mathrm{mm} \mathrm{HP}$ vs $23-\mathrm{mm} \mathrm{SC}$; and 23-mm HP vs $23-\mathrm{mm}$ SC).

As a third step, the Wilcoxon rank test for dependent samples was applied, accounting for variation from both observation times within each of the 4 valve types.
All data were expressed as mean \pm standard deviation (SD). Confidence intervals were calculated at $95 \%$ of significance.

\section{Results}

A total of 140 patients were enrolled in the study. Their demographics and clinical characteristics are shown in Table 1. Of these, 5 patients $(3.5 \%)$ died at operation (30day mortality); causes of death were low output syndrome in 4 patients and renal failure in 1 patient. There was an 


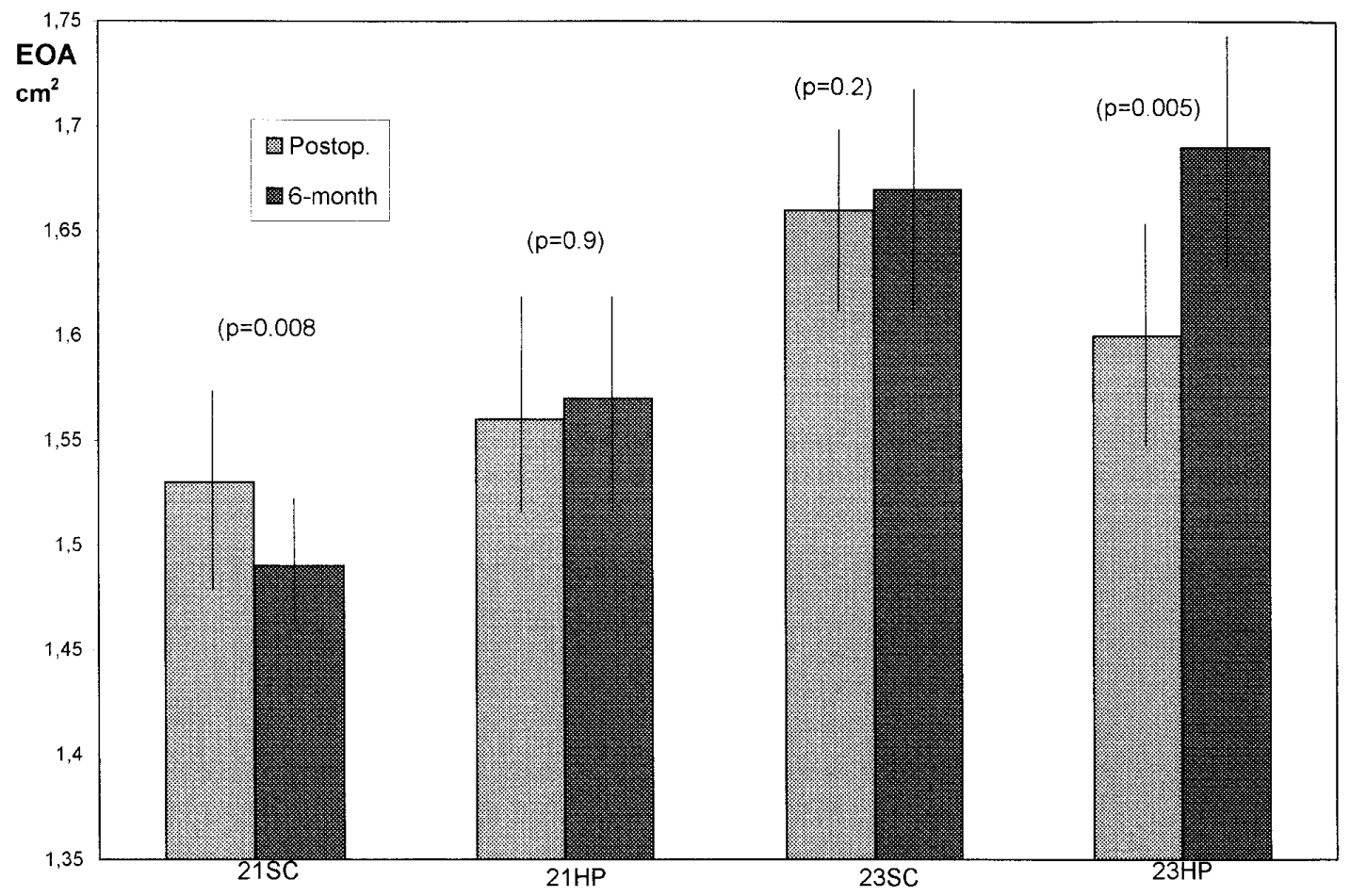

Figure 2. Serial changes of valve effective orifice areas (EOA) over time within the 4 groups. The $P$ values express the comparisons between postoperative and 6-month observation times.

TABLE 3. Six-month echocardiographic data

\begin{tabular}{|c|c|c|c|c|c|c|c|}
\hline Parameter & $\begin{array}{c}21-\mathrm{mm} \mathrm{SC} \\
(\mathrm{n}=27)\end{array}$ & $P^{*}$ & $\begin{array}{c}21-\mathrm{mm} \text { HP } \\
(\mathrm{n}=35)\end{array}$ & $P \dagger$ & $\begin{array}{c}23-\mathrm{mm} \text { SC } \\
(\mathrm{n}=30)\end{array}$ & $P \neq$ & $\begin{array}{c}23-\mathrm{mm} \text { HP } \\
(\mathrm{n}=33)\end{array}$ \\
\hline $\mathrm{CO}(\mathrm{L} / \mathrm{m})$ & $5.43 \pm 1.6$ & .14 & $4.66 \pm 1.9$ & .0022 & $6.17 \pm 1.9$ & .011 & $4.82 \pm 1.5$ \\
\hline $\mathrm{EF}$ & $0.58 \pm 0.8$ & .17 & $0.62 \pm 0.8$ & .53 & $0.66 \pm 0.6$ & .19 & $0.57 \pm 0.9$ \\
\hline Peak gradient $(\mathrm{mm} \mathrm{Hg})$ & $24.6 \pm 6.9$ & .0003 & $16.3 \pm 8.4$ & .0001 & $24.7 \pm 9.5$ & .0005 & $16.8 \pm 7.3$ \\
\hline Mean gradient $(\mathrm{mm} \mathrm{Hg})$ & $17.8 \pm 5.2$ & .001 & $12.6 \pm 6.5$ & .001 & $17.5 \pm 6$ & .0008 & $12.1 \pm 4.2$ \\
\hline $\mathrm{EOA}\left(\mathrm{cm}^{2}\right)$ & $1.49 \pm 0.4$ & 6 & $1.57 \pm 0.6$ & .45 & $1.67 \pm 0.5$ & .9 & $1.69 \pm 0.5$ \\
\hline $\mathrm{EAI}\left(\mathrm{cm}^{2}\right)$ & $0.87 \pm 0.2$ & .74 & $0.90 \pm 0.3$ & .62 & $0.94 \pm 0.3$ & .75 & $0.97 \pm 0.3$ \\
\hline $\mathrm{PI}\left(\mathrm{cm}^{2}\right)$ & $0.61 \pm 0.2$ & .88 & $0.6 \pm 0.2$ & .062 & $0.7 \pm 0.2$ & .05 & $0.58 \pm 0.2$ \\
\hline
\end{tabular}

Data are expressed as mean \pm standard deviation. The number of patients in each group is indicated in parentheses. $S C$, St Jude Medical standard cuff valve; HP, St Jude Medical Hemodynamic Plus valve; $C O$, cardiac output; $E F$, ejection fraction; EOA, effective orifice area; EAI, effective area index; $P I$, performance index.

${ }^{*} P$ value, $21-\mathrm{mm} \mathrm{SC}$ vs $21-\mathrm{mm} \mathrm{HP}$ valves.

† $P$ value, 21-mm HP vs $23-\mathrm{mm} \mathrm{SC}$ valves.

$\ddagger P$ value, 23-mm SC vs $23-\mathrm{mm}$ HP valves.

intraoperative complete detachment of a $23-\mathrm{mm} \mathrm{HP}$ valve sewing cuff resulting from disruption of the cuff's thread windings caused by a suture needle. The patient received a CarboMedics valve (Sulzer Carbomedics, Inc, Austin, Tex) as described elsewhere. ${ }^{4}$ In the follow-up, 1 patient with a 23-mm SC valve had acute aortic dissection 2 months after valve replacement and died at reoperation. Of the remaining 133 patients, 125 patients completed the 6-month follow-up, but 8 patients were censored because they refused to under- go evaluation at 6 months. Echocardiographic data and clinical results reported here are those obtained from the 125 patients.

\section{Echocardiography}

Postoperative and 6-month echocardiographic measurements (mean $\pm \mathrm{SD}$ ), together with $P$ values for comparisons between valve types, are presented in Tables 2 and 3, respectively. Figure 1 shows changes in peak and mean trans- 
valvular gradients between postoperative and 6-month measurements within each of the 4 groups, whereas Figure 2 depicts serial changes of effective orifice areas.

\section{Peak Gradient}

At both observation times, the 21-mm and 23-mm HP valves showed lower gradients than the SC valves of the same size. In particular, the 21-mm HP valves had lower gradients than the 23-mm SC valves. Interestingly, the 21$\mathrm{mm}$ HP valves had gradients similar to those of the $23-\mathrm{mm}$ HP valves. All gradients decreased over time in each group, and all these differences were statistically significant between the 2 observation times.

\section{Mean Gradient}

Postoperatively, the 21-mm SC and 21-mm HP valves had equal mean gradients, whereas the 23-mm HP valve had a slightly lower gradient than 23-mm SC valve. At 6 months both the 21- and 23-mm HP valves had lower gradients than the SC valves. All gradients decreased over time in each group, and all the differences reached statistical significance, except for those of the 23-mm SC valves.

\section{Effective Orifice Area}

There was not a statistically significant difference in effective orifice areas between the groups. At postoperative observation, the effective orifice areas of the 21-mm HP valves were slightly larger than those of the 21-mm SC valves and remained larger at the 6-month measurement. The $23-\mathrm{mm}$ HP and SC valves showed approximately the same effective orifice areas. The effective orifice areas of the 23-mm HP valves increased over time, and the difference between the 2 observation times was statistically significant. The effective orifice areas of the 21-mm SC valves decreased at the 6month measurement, and the difference was significant. The areas of the 23-mm SC valves increased only slightly, and the difference did not reach statistical significance.

\section{Effective Area Index}

At postoperative measurement, effective orifice area matched for all groups with respect to valve size and body surface area at postoperative and 6-month measurements. The effective area indices for the 23-mm HP valves increased at 6 months, and the difference between the 2 observations reached statistical significance $(P=.004)$. The effective area indices for the $21-\mathrm{mm}$ SC valves decreased slightly at 6 months, and the difference was significant $(P=$ .0008). The effective area indices for the $23-\mathrm{mm} \mathrm{SC}$ valves did not change across time $(P=.93)$.

\section{Performance Index}

Postoperatively, the 23-mm HP valves showed a lower performance index than the $23-\mathrm{mm} \mathrm{SC}$ valves, the differences not being statistically significant. Performance indices for the 23-mm HP valves and the 23-mm SC valves increased over time, but the difference was statistically significant only for the $23-\mathrm{mm} \mathrm{SC}$ valves $(P=.003)$. The performance index for the 21-mm HP valves did not change over time ( $P$ $=.2$ ). The performance index for the $21-\mathrm{mm} \mathrm{SC}$ valves decreased slightly at 6 months, and this difference was significant $(P=.0008)$.

\section{Follow-up}

At the end of 6 months of follow-up, no valve thrombosis, thromboembolic events, or anticoagulation-related hemorrhages were observed. All patients are well with a satisfactory quality of life.

\section{Discussion}

As in most practices nowadays, our population was largely composed of patients who required aortic valve replacement for severe calcified valve stenosis. Despite a reduced cuff thickness, all surgeons found the St Jude Medical HP valve relatively easy to implant in the supraannular position.

Peak and mean transvalvular gradients are still the parameters most frequently used to characterize a prosthetic heart valve. ${ }^{5}$ In our comparison with the St Jude Medical SC valves of the same outer diameter, both the 21-mm and 23-mm HP valves demonstrated much lower peak and mean transvalvular gradients both postoperatively and at 6 months after the operation. Furthermore, the 21-mm HP valves showed gradients lower than those of the 23-mm SC valves. The 21-mm valves had equal mean gradients at discharge. At 6 months the 21-mm HP valve had peak and mean gradients similar to those of the 23-mm HP valves, thereby showing the most beneficial size/gradient relationship.

Doppler echocardiography is the most commonly used method of follow-up for patients with prosthetic heart valves. ${ }^{6}$ Measurements of pressure gradients across different types of bioprosthetic and mechanical valves are reported to correlate well with catheter-derived gradients. ${ }^{5}$ Although bileaflet valves have a central flow and should allow an accurate measurement of pressure gradients, the presence of pressure recovery and high localized pressure gradients might cause an overestimation of gradients compared with the catheter-derived pressure gradient as found by Baumgartner and associates ${ }^{7}$ in St Jude Medical valves. Therefore, we should be aware the Doppler-defined gradients of our study could be slight overestimations of catheterderived gradients. With respect to gradient variations between postoperative and 6-month time points, decreases were observed within each group and were more enhanced for the 21-mm HP valves.

With regard to effective orifice area, slightly (but not significantly) larger valve areas were found for the HP valves 
than for SC valves at 6 months. In our series, the effective orifice areas of 21-mm HP valves at 6 months were smaller than those reported by Carrel and associates. ${ }^{8}$ In vivo effective orifice areas of 23-mm HP valves have not yet appeared in the literature, to the best of our knowledge. Another interesting finding of our study was the proven increase of valve areas over time for 23-mm HP valves. On the other hand, the effective orifice areas of 21-mm and 23-mm SC valves did not increase over time. All observed increases developed quite rapidly, within 6 months after operation. This observation contrasts with the findings of Henneke, Pongratz, and Bachmann, ${ }^{9}$ who noted that a postoperative interval of less than 1 year did not alter the Doppler-derived measurements of effective orifice area.

With regard to effective area indices, the 23-mm HP valve indices were higher than those of $23-\mathrm{mm} \mathrm{SC}$ valve indices at 6 months. On the other hand, at postoperative measurement, the effective area indices for the 21-mm HP valves were similar to those of the $21-\mathrm{mm}$ SC valves, but were higher at 6 months. The effective area indices for the 21-mm SC valves did not change across the 2 observation times. All the observed increases were a result of increases in effective orifice areas because all body surface areas remained constant at both observation times. Moreover, the body surface areas of our patients were above $1.7 \mathrm{~m}^{2}$.

On the basis of the assumption that an effective area index of $0.9 \mathrm{~cm}^{2} / \mathrm{m}^{2}$ is the requirement for a patient with an aortic prosthetic valve and that this value was calculated in patients with tilting disc and tissue valves, ${ }^{3}$ all our patients showed a satisfactory prosthesis-patient match.

Several studies have examined the impact of small aortic prostheses on long-term results. In a series of 254 patients, Kratz and others ${ }^{10}$ implanted $19-\mathrm{mm}$ and $21-\mathrm{mm}$ valves in 115 patients. In comparison with the overall results, the implantation of a small aortic valve was followed by a somewhat higher incidence of late sudden death in patients with body surface areas of $1.9 \mathrm{~m}^{2}$. According to Czer and associates, ${ }^{11}$ implantation of smaller valves did not affect short-term hospital results. However, in this series, results were limited to patients with body surface areas of less than $1.7 \mathrm{~m}^{2}$. Jaffe, ${ }^{12}$ Burkhardt, ${ }^{13}$ and their colleagues have suggested that peak aortic pressure gradients up to $40 \mathrm{~mm} \mathrm{Hg}$ did not bear any clinical significance for up to a decade in the majority of patients.

Performance indices were almost comparable between the groups. Our results of 0.56 to $0.71 \mathrm{~cm}^{2}$ were similar to those of other reports on St Jude Medical valves. ${ }^{8}$ Furthermore, a performance index between 0.37 and 0.44 $\mathrm{cm}^{2}$ has been reported for small Björk-Shiley valves (Shiley, Inc, Irvine, Calif), and a performance index of $0.53 \mathrm{~cm}^{2}$ has been reported for the 21-mm Medtronic-Hall mechanical valve (Medtronic, Inc, Minneapolis, Minn). ${ }^{14}$ Time-related increases in performance indices were observed for the 21- $\mathrm{mm}$ HP valves. As with effective area indices, these increases were due to improvement in effective orifice areas over time.

During the early follow-up of the 125 patients who completed the study, no valve-related morbidity or mortality was observed. Therefore, the results of our study with the St Jude Medical HP valve in the aortic position confirm the satisfactory results obtained with St Jude Medical SC valves. $^{12,13}$

All the parameters we considered were the same parameters presented in several studies on heart valve prostheses. ${ }^{6-8,14-16}$ Furthermore, our study was the first with a multicenter, controlled and randomized protocol that was carried out on the St Jude Medical HP aortic valve. Nonetheless, there may be bias in our study. Differences in cardiac output were observed that may have caused the difference in transvalvular gradients. Cross-sectional areas of the valves were measured with an edge-to-edge method, and in patients with a hypertrophic ventricular septum this may reduce effective orifice areas.

Therefore, several conclusions can be drawn from our study. St Jude Medical 21-mm and 23-mm HP valves have demonstrated better hemodynamic characteristics than St Jude Medical SC valves. These positive results confirm laboratory findings of lower pressure drops and energy losses for St Jude Medical HP valves. ${ }^{1}$ All the considered parameters demonstrated, at both observation times, that performances of the 21-mm and 23-mm HP valves were substantially better than those of the $\mathrm{SC}$ valves of the same diameters and were comparable with those of the SC valves with larger external diameters. Both a more enhanced decrease of transvalvular pressure gradients and a more enhanced increase of valve areas were observed for the HP valves versus the $\mathrm{SC}$ valves across observation times.

In particular, the 21-mm HP valves showed low gradients, even similar to those of 23-mm HP valves. With this in mind, the 21-mm HP valve may be considered a very satisfactory prosthetic valvular device in the small aortic anulus. Implantation of a 21-mm HP valve should avoid forcing a larger valve into a tight anulus or spare an aortic anulus enlargement. The use of the 21-mm HP valve would ease the procedure and reduce mortality and morbidity related to the longer operative time.

Results of early follow-up with the St Jude Medical HP valves were excellent. Nevertheless, a longer follow-up is desirable to confirm this outcome.

\section{References}

1. Fisher J. Comparative study of the hydrodynamic function of the size $19 \mathrm{~mm}$ and $21 \mathrm{~mm}$ St. Jude Medical Hemodynamic Plus bileaflet heart valves. J Heart Valve Dis. 1994;3:75-80.

2. Edmunds LH, Clark RE, Cohn LH, Grunkemeier GL, Miller DG, Weisel RD. Guidelines for reporting morbidity and mortality after cardiac valvular operations. Ann Thorac Surg. 1996;62:932-5. 
3. Dumesnil JG, Honos GN, Lemieux M, Beauchemin J. Validation and applications of indexed aortic prosthetic valve areas calculated by Doppler echocardiography. J Am Coll Cardiol. 1990;16:637-43.

4. Vitale N, Serena D, Fondacone C, Agnino A, Cicala P, de Luca T.S. L. Complete intraoperative dislodgment of a St. Jude Medical Hemodynamic Plus valve sewing cuff. J Thorac Cardiovasc Surg. 1996;112:1669-71.

5. Franzen SF, Huljebrandt IE, Konstantinov IE, Nylander E, Olin CL. Aortic valve replacement for aortic stenosis in patients with small aortic root. J Heart Valve Dis. 1996;5(Suppl III):S284-8.

6. Chambers J. Echocardiography and the small aortic root. J Heart Valve Dis. 1996;5(Suppl III):S264-8.

7. Baumgartner H, Khan S, DeRobertis M, Czer L, Maurer G. Effect of prosthetic valve design on the Doppler-catheter gradient correlation: an in vitro study of normal St. Jude, Medtronic-Hall, Starr-Edwards and Hancock valves. J Am Coll Cardiol. 1992;19:324-32.

8. Carrel T, Zingg U, Aeschbacher B, Turina MI. Early in vivo experience with the Hemodynamic Plus St. Jude Medical heart valves in patients with narrowed aortic annulus. Ann Thorac Surg. 1996;61:1418-22.

9. Henneke KH, Pongratz G, Bachmann L. Limitations of Doppler echocardiography in the assessment of prosthetic valve hemodynamics. J Heart Valve Dis. 1995;4:18-25.

10. Kratz JM, Sade RM, Crumbley AJ, Stroud MR The risk of small St. Jude aortic valve prostheses. Ann Thorac Surg. 1994;57:114-9.

11. Czer LSC, Chaux A, Matloff JM, DeRobertis MA, Nessim SA, Scarlata D, et al. Ten-year experience with the St. Jude Medical valve for primary valve replacement. J Thorac Cardiovasc Surg. 1990;100:44-55.

12. Jaffe WM, Coverdale HA, Roche AH, Whitlock RM, Neutze JM, Barratt-Boyes GB. Rest and exercise hemodynamics of 20 to $23 \mathrm{~mm}$ allograft, Medtronic Intact (porcine), and St. Jude Medical valves in the aortic position. J Thorac Cardiovasc Surg. 1990;100:167-74.

13. Burkhardt D, Hoffman A, Vogt S, Roth J, Pfisterer M, Weiss P, et al. Heart valve replacement with St. Jude Medical valve prosthesis: longterm experience in 743 patients. Circulation. 1988;78(Suppl):I-18-24.

14. Aberg H, Holmgren A. Hemodynamic evaluation of the Convexo-concave Björk-Shiley prosthesis in patients with narrow aortic annulus. Scand J Thorac Cardiovasc Surg. 1981;15:111-6.

15. De Paulis R, Sommariva L, Russo F, Tomai F, Tondo A, Pagliaricci C, et al. Doppler echocardiography evaluation of the CarboMedics valve in patients with small aortic anulus and valve prosthesis-body surface area mismatch. J Thorac Cardiovasc Surg. 1994;108:57-62.
16. De Paulis R, Sommariva L, De Matteis GM, Polisca P, Tomai F, Bassano C, et al. Hemodynamic performances of small diameter CarboMedics and St. Jude valves. J Heart Valve Dis. 1996;5(Suppl III):S339-443

\section{Appendix: The Italian Multicenter Study Group on the St Jude Medical HP Aortic Valve Prosthesis}

Department of Cardiac Surgery, University of Bari: Nicola Vitale, Luigi de Luca T.S. (surgeons), Giustina Castellaneta (echocardiographer). Dr Vitale was a PhD student in Cardiac Surgical Sciences at the Department of Cardiac Surgery, Second University of Naples.

Department of Cardiac Surgery, University of Bologna: Pier Maria Mikus, Angelo Pierangeli (surgeons); Ilaria Caldarera (echocardiographer).

Department of Cardiac Surgery, University of Catania: Rocco Meduri, Mauro Abbate (surgeons); Antonio Silvia (echocardiographer).

Department of Cardiac Surgery, University of Padova: Dino Casarotto (surgeon); Giuseppe Fasoli (echocardiographer).

Department of Cardiac Surgery, University of Brescia: Claudio Muneretto (surgeon).

Department of Cardiac Surgery, University of Parma: Tiziano Gherli (surgeon); Andrea Contini (echocardiographer).

Department of Cardiac Surgery, University of Roma 1: Riccardo Sinatra, Benedetto Marino (surgeons); Nicola Alessandri (echocardiographer).

Department of Cardiac Surgery, University of Roma 2: Antonio Scafuri, Luigi Chiariello (surgeons); Luigi Sommariva (echocardiographer).

Department of Cardiac Surgery, University of Torino: Michele di Summa, Elio Di Rosa, (surgeons).

Department of Statistics, University of Cagliari: Nicola Tedesco (biostatistician). 